\title{
Efficacy of a New Nutraceutical Formulation in Patients with Symptomatic Uncomplicated Diverticular Disease (SUDD): a Prospective Observational Study
}

\author{
Ferdinando D’Amico ${ }^{1,2}$, Giulia Fiorini ${ }^{3}$, Antonio Tursi ${ }^{4}$, Ilaria Maria Saracino ${ }^{3}$, Matteo Pavoni ${ }^{3}$, Silvio Danese ${ }^{1,2}$, \\ Dino Vaira ${ }^{3}$
}

1) Department of Biomedical

Sciences, Humanitas

University, Milan, Italy

2) IBD Center, Department of Gastroenterology, Humanitas Clinical and Research Center, Milan, Italy

3) Department of Surgical and Medical Sciences, Sant'Orsola Hospital, University of Bologna, Bologna, Italy 4) Gastroenterology Service, ASL BAT, Andria, Italy

\footnotetext{
Address for correspondence: Dino Vaira, MD, $\mathrm{PhD}$

Department of Medical and Surgical Sciences, University of Bologna,

"S. Orsola" University Hospital,

Via Massarenti, 9, 40138,

Bologna, Italy

berardino.vaira@unibo.it
}

\begin{abstract}
Background \& Aim: Symptomatic uncomplicated diverticular disease (SUDD) is characterized by abdominal pain, bloating and altered bowel habits (constipation or diarrhea) attributed to diverticula in the absence of macroscopic mucosal alterations. There is no consensus about management of these patients. DIVER-100", an association of natural active ingredients may be effective in the treatment of patients with SUDD. The aim was to evaluate the efficacy and safety of DIVER-100 in patients with SUDD.

Methods: We conducted a prospective observational study to evaluate the efficacy of DIVER- $100^{\circ}$ in consecutive patients with SUDD, confirmed by radiology or endoscopy. All patients were treated with DIVER- $100^{\circ} 2$ capsules/day 10 days per month, for 3 months. The primary endpoint was the clinical remission rate, defined as the reduction of abdominal pain and bloating, improvement of bowel habits and prevention of acute diverticulitis (AD). The secondary endpoint was the rate of adverse events.

Results: One hundred and one patients were consecutively enrolled at the Internal Medicine and Gastroenterology Unit, Sant'Orsola Hospital, Bologna, Italy. DIVER- $100^{\circ}$ was effective in inducing remission of symptoms in 12 patients (11.9\%) at 3 months and in 10 patients (9.9\%) at 6 months. DIVER- $100^{\circ}$ significantly reduced abdominal pain and bloating in $45.5 \%$ and $57.4 \%$ of patients respectively ( $\mathrm{p}<0.001$ ) after 3 months. No episodes of $\mathrm{AD}$ and no adverse events related to DIVER- $-100^{\circ}$ were recorded at month 6 in the study population.

Conclusions: DIVER- $100^{\circ}$ is a safe and effective nutraceutical compound in obtaining remission and symptom relief in SUDD patients. Further randomized, placebo-controlled clinical trials are needed to confirm these preliminary data.
\end{abstract}

Key words: SUDD - diverticular disease - DICA 1 - nutraceuticals - DIVER-100.

Abbreviations: AD: acute diverticulitis; DD: diverticular disease; SUDD: symptomatic uncomplicated diverticular disease; DICA: Diverticular Inflammation and Complications Assessment.

\section{INTRODUCTION}

Diverticular disease (DD) is one of the most frequent gastroenterological diseases in western countries and the most frequent finding on colonoscopies [1]. Approximately 20\% of patients with diverticula develop symptoms, which can range from symptomatic uncomplicated diverticular disease (SUDD) to acute diverticulitis (AD) and its complications (perforation, peritonitis, fistulas, abscesses) [2]. SUDD is a syndrome characterized by left lower abdominal pain, bloating and altered bowel habits, including constipation or diarrhea, attributed to diverticula in the absence of macroscopic mucosal alterations [3]. Pathogenesis of SUDD is not clear, although genetic susceptibility, diet, neuromuscular dysfunction, altered gut microbiome and chronic mucosal inflammation play a key role, thus representing an attractive therapeutic target [4].

Rifaximin, mesalazine and probiotics are the most frequently used drugs for the treatment of patients with SUDD. A metaanalysis, which included 4 prospective randomized trials and over 1600 patients showed the efficacy of rifaximin in the treatment of symptoms in patients with SUDD [5]. A recent multicenter retrospective study confirmed the efficacy of rifaximin in the treatment of SUDD symptoms over a follow-up period of 8 years [6]. A systematic review with meta-analysis of randomized, placebo controlled trials, enrolling 379 patients 
demonstrated a statistically significant relief of symptoms, as well as a lower rate of $\mathrm{AD}$ onset in patients treated with mesalazine, compared to those treated with placebo ( $80 \%$ vs $62.7 \%$ and $19.3 \%$ vs $33.3 \%$ respectively) [7]. A systematic review by Lahner et al. [8] showed an apparent trend to a positive clinical response on abdominal symptoms and their recurrence in patients with SUDD treated with probiotics.

Recently, clinical studies showed that the use of nutraceutical compounds, reducing the inflammatory response and intestinal permeability, might play a role in the treatment of these patients. However, despite the different therapeutic possibilities, there is no consensus about management of SUDD patients.

DIVER-100 ${ }^{\circledR}$ is a new association of natural active ingredients containing Boswellia serrata, inulin, niacin, cranberry, vitamin B1, B2, B6, B12, zinc and folic acid.

The aim of this prospective open label trial was to evaluate the efficacy and safety of DIVER- $100^{\circ}$ for the treatment of SUDD patients.

\section{METHODS}

We aimed to evaluate prospectively the efficacy of DIVER- $100^{\infty}$ in inducing remission of symptoms in consecutive adult patients affected by SUDD, confirmed by radiology (computer tomography) or by colonoscopy. The study included only patients scored as DICA 1 according to the Diverticular Inflammation and Complications Assessment (DICA) classification [9]. All patients were treated with DIVER-100 ${ }^{\circ}$ 2 capsules/day 10 days per month, for 3 months. Exclusion criteria were: $\mathrm{AD}$, complicated $\mathrm{DD}$ (i.e. perforation, bleeding), concomitant unstable comorbid conditions (i.e. chronic renal failure, liver cirrhosis), active malignant neoplasm, co-existing inflammatory bowel disease, pregnancy or lactation, treatment with antibiotics or NSAIDs in the 30 days prior to enrollment, inability to sign an informed consent.

Patients were assessed at baseline and at 3 and 6 months after the beginning of the treatment regarding their general condition, symptoms and adverse events. The primary endpoint of the study was the clinical remission rate, defined as the disappearance of abdominal pain and bloating, improvement of bowel habits and prevention of AD. This endpoint was assessed using a visual analog (VAS) scale (a 10-point visual scale, assigning numerical values of 0 for absence of symptom until 10 for the most severe symptom). The secondary endpoint was the rate of adverse events.

\section{Statistical analysis}

Frequency, distribution analyses and non-parametric tests were used for the statistical analysis. Results were considered statistically significant for $\mathrm{p}$ values $<0.05$.

\section{RESULTS}

One hundred and one patients were consecutively enrolled at the Internal Medicine and Gastroenterology Unit, Sant'Orsola Hospital, Bologna, Italy. Patient characteristics are summarized in Table I.

DIVER- $100^{\circ}$ was effective in inducing remission of symptoms (VAS score: 0 ) in 12 patients (11.9\%) at 3 months
TableI. Patient characteristics

\begin{tabular}{lc}
\hline Patient characteristics & $\mathrm{n}(\%)$ \\
\hline Enrolled patients & 101 \\
Male & $54(53.4 \%)$ \\
Median age & 67 years (range $35-89$ years) \\
Location of the diverticula: & $86(85.1 \%)$ \\
- Sigmoid region• & $2(2 \%)$ \\
• Sigmoid region, descending and & \\
transverse colon & $1(1 \%)$ \\
- Descending colon & $1(1 \%)$ \\
- Ascending colon & $11(10.9 \%)$ \\
- Descending and ascending colon & \\
Number of diverticula: & $30(29.7 \%)$ \\
$>15$ diverticula & \\
Comorbidities: & $56(55.4 \%)$ \\
- Cardiovascular diseases & $5(4.9 \%)$ \\
- Rheumatological diseases & $2(2 \%)$ \\
- Metabolic diseases & $17(16.8 \%)$ \\
\hline
\end{tabular}

and in 10 patients $(9.9 \%)$ at 6 months. DIVER- $100^{\circledR}$ was already able after 3 months to significantly reduce abdominal pain in $45.5 \%$ of patients (VAS score from 3.028 to $1.631, \mathrm{p}<0.001$ ) and bloating in $57.4 \%$ of patients (VAS score from 4.560 to 3.021 , $\mathrm{p}<0.001)$. After 6 months, a further, statistically significant reduction in abdominal pain (VAS score from 3.028 to 1.128) and bloating (VAS score from 4.560 to 2.485 ) was confirmed in $46.5 \%$ and in $58.4 \%$ respectively of patients treated with DIVER- $100^{\oplus}$ ( $\mathrm{p}<0.001$ for both comparisons). An improvement in bowel movements, not statistically significant, was recorded in 26 patients $(25.7 \%)$ at 3 -month follow-up and in 21 patients (20.8\%) a 6-month follow-up. The efficacy data of DIVER-100 are shown in Fig 1.

No episodes of $\mathrm{AD}$ and no adverse events related to DIVER- $100^{\circ}$ were recorded in the 6 months of follow-up in the study population. No association was found between remission and baseline characteristics (comorbidities, concomitant medication, location and number of diverticula) of patients (all $\mathrm{p}>0.05$ ).

\section{DISCUSSION}

SUDD is generally characterized by mild to moderate symptoms, which significantly impair the patients' quality of life [10]. The goal of treatment is to reduce symptoms and prevent the onset of $\mathrm{AD}$.

Conflicting data regarding the efficacy of commonly used drugs for the treatment of SUDD such as rifaximin and mesalazine make DD an attractive challenge for gastroenterologists. The main doubts on the use of rifaximin are related to the risk of adverse events and allergic reactions and to the possible antibiotic resistance [11]. For mesalazine, on the other hand, there are no clear data to confirm its effectiveness in determining symptom relief in SUDD patients [12].

From these conflicting data arises the unmet need for new therapeutic agents, which by acting with different and combined mechanisms would control the symptoms. 


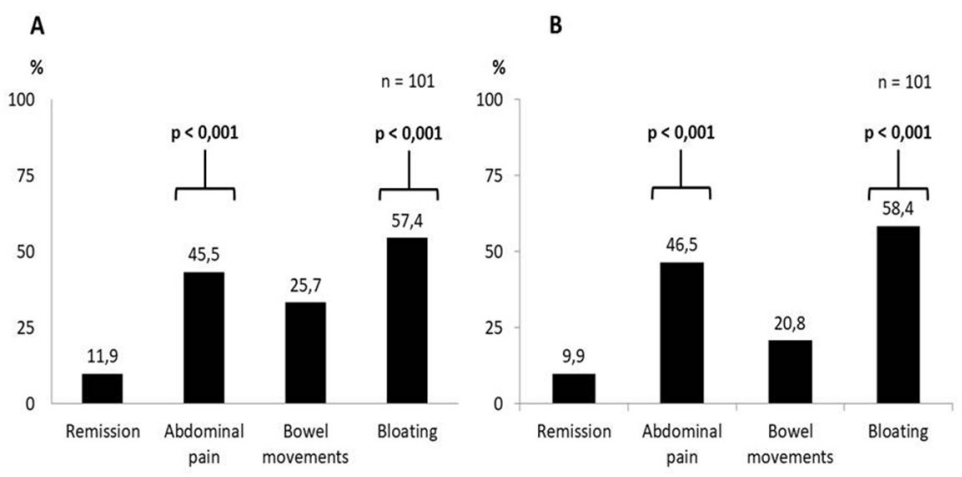

Fig. 1. Efficacy of DIVER- $100^{\circledR}$ at 3 months (A) and at 6 months (B)

Our study showed that DIVER-100', a new nutraceutical formulation, is effective in determining the remission of symptoms in patients with SUDD. Furthermore, it has an excellent safety profile as suggested by the lack of adverse events in the study population. Moreover, the efficacy of DIVER- $100^{\circ}$ on the reduction of abdominal pain and bloating seemed not only rapid, showing a considerable and statistically significant benefit at the end of 3 months of treatment, but also lasting, as patients presented a relief of symptoms even 3 months after the end of therapy. We do not know the pathophysiological mechanism that determines the continuation of the effect during follow up, but it could probably be related to the inhibition of inflammation and to the activity on the gut microbiota which determines a benefit that lasts over time.

The efficacy and safety of this nutraceutical compound could be explained by the combination of the various ingredients that compose it. Boswellia serrata and zinc, as investigated in vitro and in animal experimental models of intestinal inflammation, can preserve the intestinal epithelial barrier and function, and protect from oxidative and inflammatory damages $[13,14]$. Cranberries, reducing the production of biofilm and the adhesion capacity of micro-organisms, have an anti-inflammatory effect [15]. Prebiotics such as inulin are nondigestible food ingredients which are fermented at the level of the colon, causing changes in the intestinal microbiota and resulting in beneficial effects [16]. Probiotics competing with pathogenic micro-organisms could lead to healthy benefits, reducing colonization and bacterial overgrowth of the gastrointestinal tract and favoring the balance of the gut microbiota [17].

Moreover, recently a 12 -month, prospective, pilot study, including 15 patients with SUDD (14 DICA 1 patients and 1 DICA 2 patient) showed that DIVER- $100^{\circ}$ was effective to reduce abdominal pain, meteorism, constipation and diarrhea. No adverse event was reported during the 12 month-follow up [18].

Despite these promising preliminary data, further randomized and placebo-controlled clinical trials are needed to confirm the efficacy and safety data of DIVER- $100^{\circledR}$ and to rule out a possible placebo effect related to its intake.

\section{CONCLUSION}

DIVER- $100^{\circ}$ was effective and safe in inducing remission of symptoms in SUDD patients and about half of patients treated with this new formulation had significant relief of abdominal pain and bloating after 3 months of therapy. Further randomized and placebo-controlled clinical trials are needed to confirm these preliminary data. Finally, comparative and/or combination trials between drugs commonly used for the treatment of SUDD and new nutraceutical formulations could provide additional data to optimize patient treatment and to define a therapeutic algorithm.

Conflicts of interest: S.D.: consultancy fees from AbbVie, Allergan, Amgen, AstraZeneca, Biogen, Boehringer Ingelheim, Celgene, Celltrion, Ferring Pharmaceuticals Inc., Gilead, Hospira, Janssen, Johnson \& Johnson, MSD, Mundipharma, Pfizer, Roche, Sandoz, Takeda, TiGenix, UCB, and Vifor. The other authors declare that they have no conflict of interest.

\section{REFERENCES}

1. Tursi A. Diverticulosis today: unfashionable and still underresearched. Therap Adv Gastroenterol 2016;9:213-228. doi:10.1177/1756283X15621228

2. Tursi A, Papagrigoriadis S. Review article: the current and evolving treatment of colonic diverticular disease. Aliment Pharmacol Ther 2009;30:532-546. doi:10.1111/j.1365-2036.2009.04072.x

3. Scarpignato C, Barbara G, Lanas A, Strate LL. Management of colonic diverticular disease in the third millennium: Highlights from a symposium held during the United European Gastroenterology Week 2017. Therap Adv Gastroenterol 2018;11:1756284818771305. doi:10.1177/1756284818771305

4. Lué A, Laredo V, Lanas A. Medical Treatment of Diverticular Disease: Antibiotics. J Clin Gastroenterol 2016;50 Suppl 1:S57-S59. doi:10.1097/ MCG.0000000000000593

5. Bianchi M, Festa V, Moretti A, et al. Meta-analysis:long-term therapy with rifaximin in the management of uncomplicated diverticular disease. Aliment Pharmacol Ther 2011;33:902-910. doi:10.1111/j.13652036.2011.04606.x

6. Di Mario F, Miraglia C, Cambiè G, et al. Long-term efficacy of rifaximin to manage the symptomatic uncomplicated diverticular disease of the colon. J Investig Med 2019;67:767-770. doi:10.1136/jim-2018-000901

7. Picchio M, Elisei W, Tursi A. Mesalazine to treat symptomatic uncomplicated diverticular disease and to prevent acute diverticulitis occurrence. A systematic review with meta-analysis of randomized, placebo-controlled trials. J Gastrointestin Liver Dis 2018;27:291-297. doi:10.15403/jgld.2014.1121.273.pic

8. Lahner E, Bellisario C, Hassan C, Zullo A, Esposito G, Annibale B. Probiotics in the Treatment of Diverticular Disease. A Systematic 
Review. J Gastrointestin Liver Dis 2016;25:79-86. doi:10.15403/ jgld.2014.1121.251.srw

9. Tursi A, Brandimarte G, Di Mario F, et al. Development and validation of an endoscopic classification of diverticular disease of the colon: the DICA classification. Dig Dis 2015;33:68-76. doi:10.1159/000366039

10. Comparato G, Fanigliulo L, Aragona G, et al. Quality of life in uncomplicated symptomatic diverticular disease: is it another good reason for treatment? Dig Dis 2007;25:252-259. doi:10.1159/000103896

11. Cuomo R, Barbara G, Annibale B. Rifaximin and diverticular disease:Position paper of the Italian Society of Gastroenterology (SIGE). Dig Liver Dis 2017;49:595-603. doi:10.1016/j.dld.2017.01.164

12. Iannone A, Ruospo M, Wong G, et al. Mesalazine for People with Diverticular Disease: A Systematic Review of Randomized Controlled Trials. Can J Gastroenterol Hepatol 2018;2018:5437135. doi:10.1155/2018/5437135

13. Catanzaro D, Rancan S, Orso G, et al. Boswellia serrata Preserves Intestinal Epithelial Barrier from Oxidative and Inflammatory Damage. PLoS One 2015;10:e0125375. doi:10.1371/journal.pone.0125375
14. Sturniolo GC, Fries W, Mazzon E, Di Leo V, Barollo M, D’inca R. Effect of zinc supplementation on intestinal permeability in experimental colitis. J Lab Clin Med 2002;139:311-315. doi:10.1067/ mlc.2002.123624

15. Blumberg JB, Basu A, Krueger CG, et al. Impact of Cranberries on Gut Microbiota and Cardiometabolic Health: Proceedings of the Cranberry Health Research Conference 2015. Adv Nutr 2016;7:759S-770S. doi:10.3945/an.116.012583

16. Akram W, Garud N, Joshi R. Role of inulin as prebiotics on inflammatory bowel disease. Drug Discov Ther 2019;13:1-8. doi:10.5582/ddt.2019.01000

17. Sullivan A, Nord CE. Probiotics and gastrointestinal diseases. J Intern Med 2005;257:78-92. doi:10.1111/j.1365-2796.2004.01410.x

18. Tursi A, Brandimarte G, Di Mario F, Elisei W, Picchio M. Efficacy and safety of a new nutraceutical formulation in managing patients with symptomatic uncomplicated diverticular disease: a 12-month prospective, pilot study. J Gastrointestin Liver Dis 2018;27:201-202. doi:10.15403/jgld.2014.1121.272.fef 